\title{
Superhydrophobic Coating Derived from the Spontaneous Orientation of Janus Particles
}

Tiantian Yang,${ }^{\S}{ }^{\dagger}$ Yuanyuan Li,,${ }^{\S} \dagger$ Haoguan Gui, ${ }^{\star}$ Deming Du, ${ }^{\star}$ Yi Du, ${ }^{\star}$ Xi-Ming Song ${ }^{* \dagger}$ and Fuxin Liang ${ }^{*}$

${ }^{\text {a }}$ Liaoning Provincial Key Laboratory for Green Synthesis and Preparative Chemistry of Advanced Materials, Liaoning University, Shenyang 110036, China

${ }^{\mathrm{b}}$ Department of Chemical Engineering, Tsinghua University, Beijing 100084, China.

Corresponding authors:

songlab@lnu.edu.cn (Prof. Song)

liangfuxin@tsinghua.edu.cn (Prof. Liang)

$\S$ T.Y. and Y.L. contributed equally to this work.

Experimental section

Figure $\mathrm{S} 1 \sim \mathrm{S} 9$

Movie S1 S4 


\section{Supporting Figures}

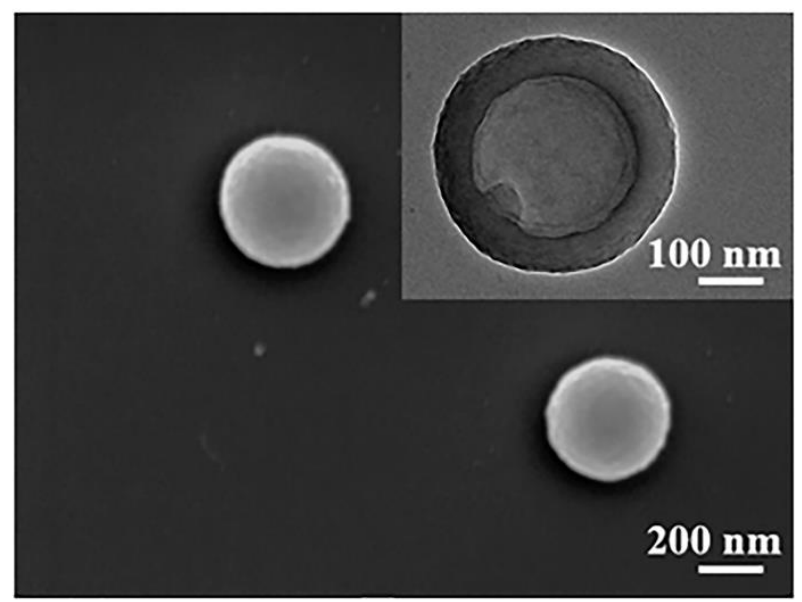

Figure S1. SEM image of PDVB/PS Seeds.
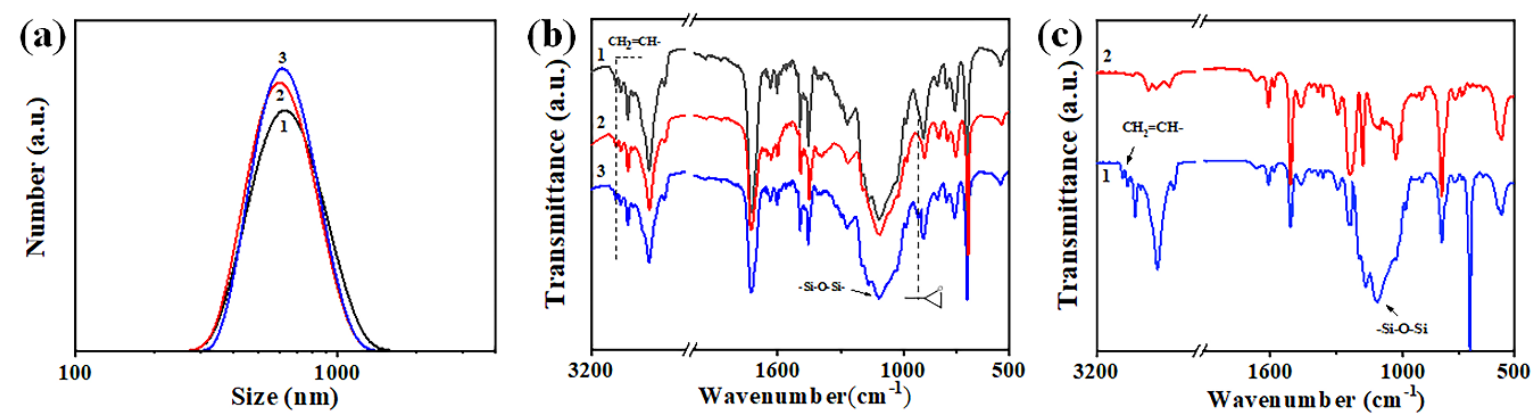

Figure S2. (a) DLS analysis and (b) FT-IR spectra of the representative JPs: 1) the silica@PDVB/PS JP, 2) the silica@PDVB/PS JP and 3) the epoxy-silica@PDVB/PS JP; (c) FT-IR spectra of the representative JPs coating after immersing in acetone for $24 \mathrm{~h}: 1$ ) the epoxy-silica@PDVB/PS JP, 2) the silica@PDVB/PS JP.
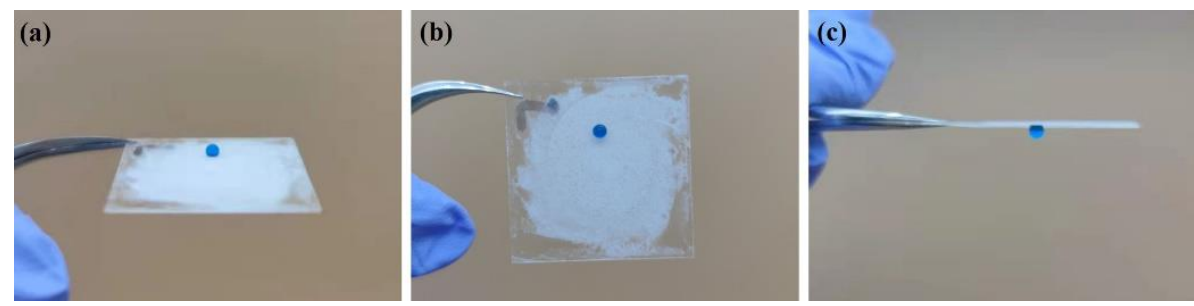

Figure S3. Water droplets contour on the composite coating with silica@PDVB JPs after the 
substrate is tilt at varied angles: (a) $0^{\circ}$; (b) $90^{\circ}$; (c) $180^{\circ}$.

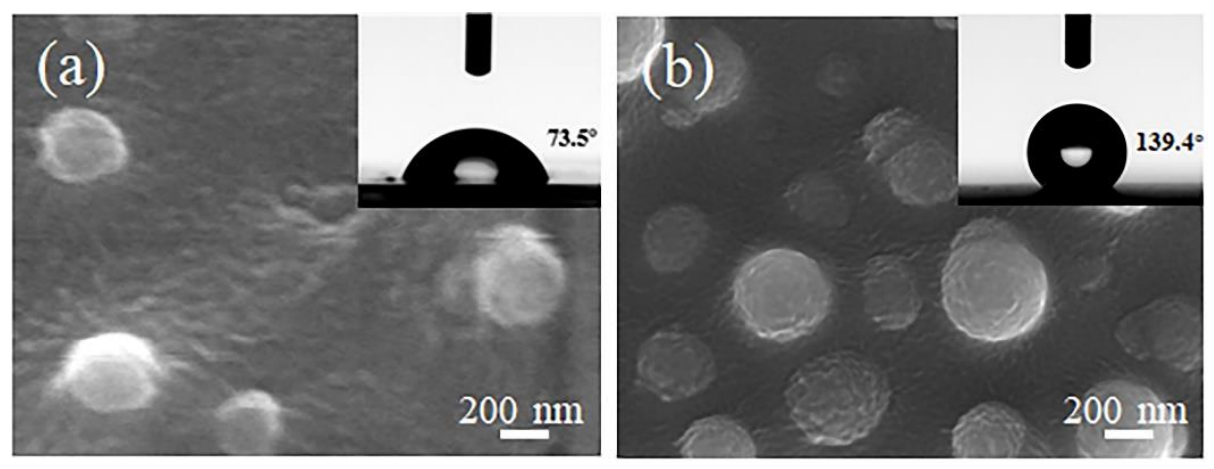

Figure S4. SEM images and WCA images of the coatings with (a) $10 \mu \mathrm{L}$, (b) $70 \mu \mathrm{L}$ epoxysilica@PDVB JPs dispersion.

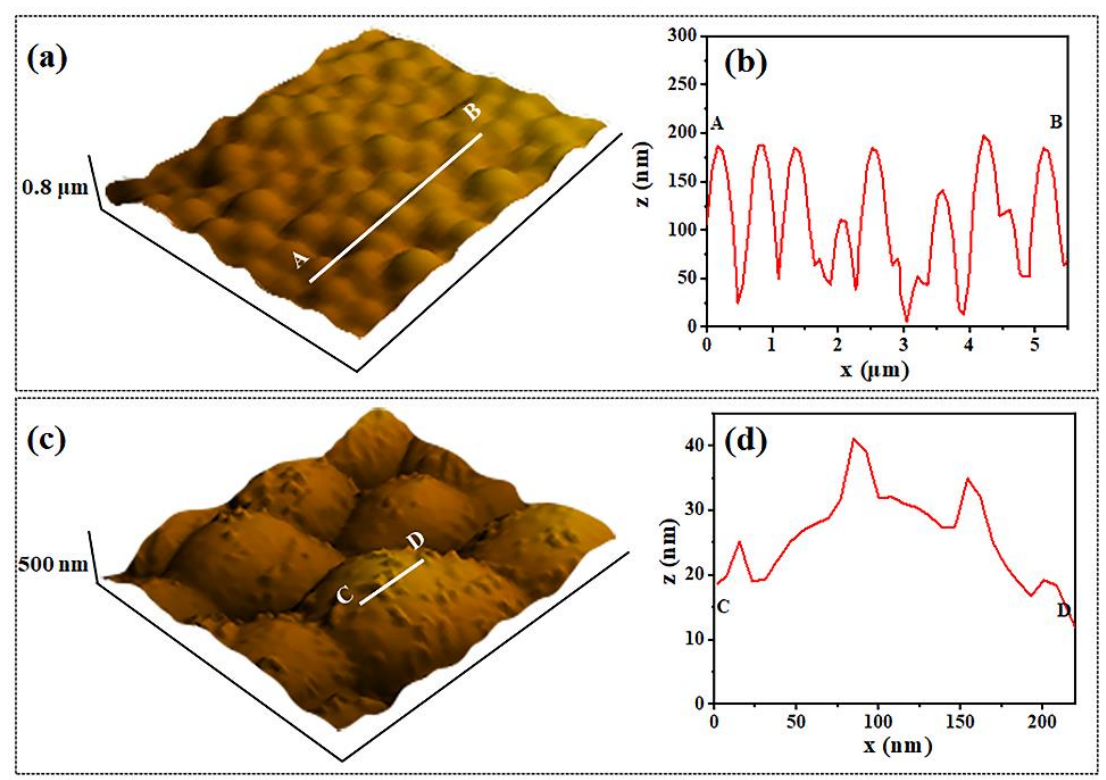

Figure S5. The surface roughness of the epoxy-silica@PDVB JPs coating: (a) the AFM image in low magnification and (b) the altitude variation from point A to point B; (c) the AFM image in high magnification and (d) the altitude variation from point $\mathrm{C}$ to point $\mathrm{D}$. 

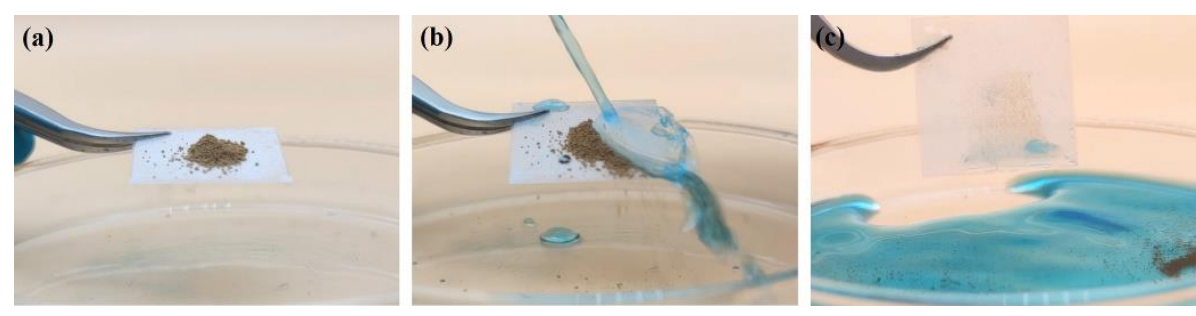

Figure S6. Flushing the pollutional silica@ PDVB JPs composite coating: (a) dirty coating, (b) flushing and (c) unclean coating.
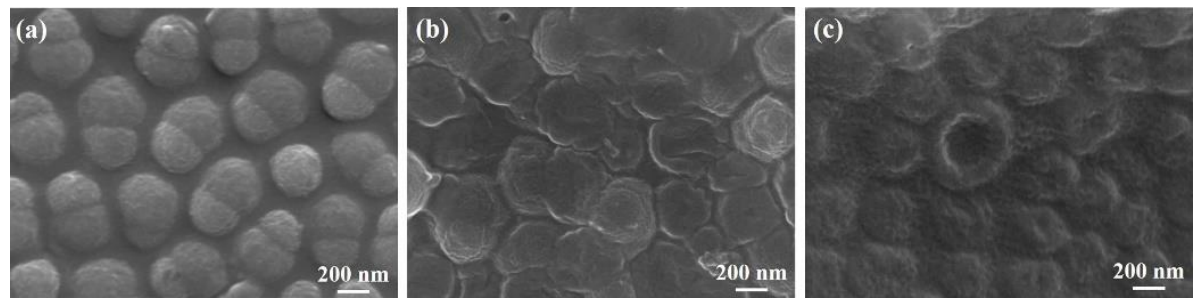

Figure S7. SEM images of the silica@PDVB JPs composite coating after immersing in (a) ethanol, (b) acetone and (c) toluene for $24 \mathrm{~h}$.

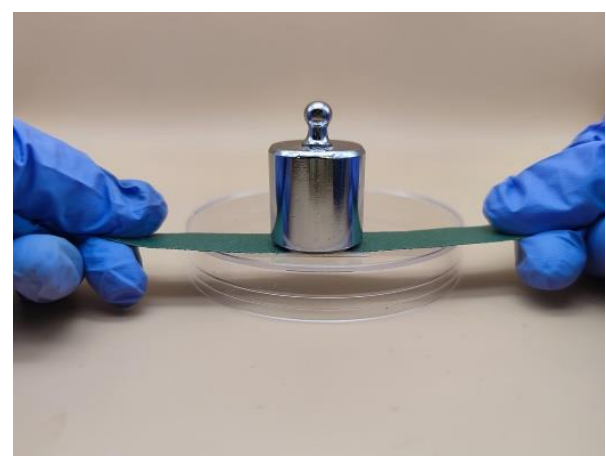

Figure S8. The digital photograph of the abrasion experiment. 

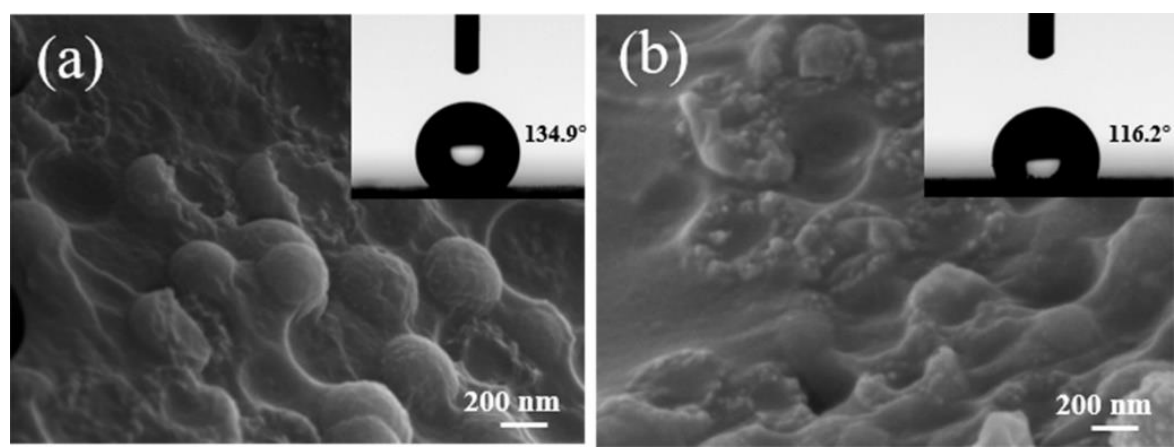

Figure S9. SEM and WCA images of the sample after mechanical wearing for (a) 80 cycles (b) 160 cycles. 


\section{Supporting Videos}

SI Video 1. Water droplets rolling down from a superhydrophobic coating tilted at $15^{\circ}$.

SI Video 2. Self-cleaning of the pollutional superhydrophobic coating.

SI Video 3. Flushing the pollutional silica@PDVB JPs composite coating.

SI Video 4. Water flushing $\left(\sim 8 \mathrm{~m} \cdot \mathrm{s}^{-1}\right)$ the superhydrophobic coating. 